\title{
Targeting autophagy in cancer management - strategies and developments
}

\author{
This article was published in the following Dove Press journal: \\ Cancer Management and Research \\ II September 2015 \\ Number of times this article has been viewed
}

\author{
Bulent Ozpolat' \\ Doris M Benbrook ${ }^{2}$ \\ 'Department of Experimental \\ Therapeutics, The University of \\ Texas - Houston, MD Anderson \\ Cancer Center, Houston, TX, \\ ${ }^{2}$ Department of Obstetrics and \\ Gynecology, University of Oklahoma \\ HSC, Oklahoma City, OK, USA
}

Correspondence: Bulent Ozpolat Department of Experimental Therapeutics, The University of Texas, MD Anderson Cancer Center, I90I East Road, 3SCR3.342I, Houston, TX 77054, USA Email bozpolat@mdanderson.org

\begin{abstract}
Autophagy is a highly regulated catabolic process involving lysosomal degradation of intracellular components, damaged organelles, misfolded proteins, and toxic aggregates, reducing oxidative stress and protecting cells from damage. The process is also induced in response to various conditions, including nutrient deprivation, metabolic stress, hypoxia, anticancer therapeutics, and radiation therapy to adapt cellular conditions for survival. Autophagy can function as a tumor suppressor mechanism in normal cells and dysregulation of this process (ie, monoallelic Beclin-1 deletion) may lead to malignant transformation and carcinogenesis. In tumors, autophagy is thought to promote tumor growth and progression by helping cells to adapt and survive in metabolically-challenged and harsh tumor microenvironments (ie, hypoxia and acidity). Recent in vitro and in vivo studies in preclinical models suggested that modulation of autophagy can be used as a therapeutic modality to enhance the efficacy of conventional therapies, including chemo and radiation therapy. Currently, more than 30 clinical trials are investigating the effects of autophagy inhibition in combination with cytotoxic chemotherapies and targeted agents in various cancers. In this review, we will discuss the role, molecular mechanism, and regulation of autophagy, while targeting this process as a novel therapeutic modality, in various cancers.
\end{abstract}

Keywords: autophagy inhibition, chemotherapy, tumor microenvironment

\section{Background}

Macroautophagy (conventionally called autophagy), which is complementary to the ubiquitin-proteasome system, is responsible for the vast majority of regulated protein recycling, in addition to the capture and degradation of mitochondria, Golgi complexes, polyribosomes, endoplasmic reticulum (ER), and other intracellular constituents, such as proteins, aggregates, and building blocks. ${ }^{1-4}$ The process of autophagy occurs continuously at basal levels in healthy cells to eliminate long-lived, misfolded, and damaged constituents through autolysosomes (autophagolysosome). ${ }^{2}$ Lysosomes are responsible for the degradation of extracellular macromolecules taken up by the cells through endocytosis or phagocytosis, as well as those from the cytoplasm through merging with autophagosomes.

Autophagy is characterized by the formation of double-layer membrane vesicles called autophagosomes that capture intracellular components and subsequently merge with lysosomes, leading to digestion of the cargo. ${ }^{3}$ The process is used by normal and cancer cells as mechanisms of recycling building blocks and conserving energy, in addition to eliminating toxic materials for homeostasis and survival. ${ }^{4}$

Autophagy can be selective through the formation of autophagosomes around protein aggregates or damaged organelles by actions of autophagosomal membrane 
receptors (eg, NBR1, p62/SQSTM1, Nix). ${ }^{4}$ There are two other forms of autophagy in eukaryotic cells called microautophagy and chaperone-mediated autophagy, which can also specifically target organelles, including mitochondria and intracellular molecules. ${ }^{3}$ Microautophagy, the nonselective lysosomal degradation process, is involved in the maintenance of organelle size, membrane homeostasis, and cell survival. ${ }^{5}$ Chaperone-mediated autophagy is mediated by a chaperone protein called heat shock cognate 70 (Hsc70), which binds to specific proteins containing certain amino acid sequences or motifs and targets them into the lysosome through the lysosome-associated membrane protein type 2A (LAMP2A) receptor. ${ }^{6}$ Selective removal of mitochondria, ER, or peroxisomes by autophagy is referred to as mitophagy, ERphagy, and peroxyphagy, respectively. ${ }^{7}$ Mitophagy is an important mitochondrial quality control mechanism that eliminates damaged mitochondria. ${ }^{7}$ BNIP3 is also degraded upon formation of the autophagolysosome, and thus reduction of BNIP3 is considered an indication of mitophagic and ERphagic flux. PINK1 and Parkin selectively bind to damaged mitochondria, and target these organelles for autophagic degradation through the ubiquitination of mitochondrial protein. ${ }^{7}$

Autophagy-based degradation pathways may lead to autophagy-associated cell death, which is important for development, differentiation, aging, and cellular remodeling under certain environmental stress conditions. ${ }^{8-11}$ All of these forms of autophagy can be induced by nutrient deprivation, hypoxia, and other cellular stresses including metabolic and therapeutic stress such as chemotherapy, radiation, natural polyphenolic compounds, and inhibitors of mammalian target of rapamycin (mTOR) kinase activity (ie, rapamycin)..$^{9,10}$ Thus, dysregulation of autophagy disrupts physiological processes and has been implicated in the pathogenesis of various conditions, including cancer and neurological diseases, such as Alzheimer, Parkinson's, and Huntington's diseases. ${ }^{11}$ When functioning properly, the autophagic process in neuronal cells prevents neurodegeneration by eliminating the accumulation of abnormal intracellular proteins. Thus, wellbalanced regulation of autophagic machinery is critical for prevention against some neurological diseases and cancer.

\section{Molecular mechanisms regulating autophagy}

The regulation of autophagy is an evolutionarily conserved and highly complex process, consisting of several basic phases, including 1) initiation; 2) nucleation; 3) maturation; and 4) merging with lysosomes, resulting in the degradation of sequestered material. The successive steps are regulated by about 30 autophagy-associated genes/proteins (Atg/Apg) in conjunction with various signaling pathways (Figure 1). ${ }^{12}$ The initiation phase is regulated by a complex consisting of Atg1, ULK1, and Atg13. ${ }^{13}$ This is the point where a cell's nutritional status and signaling through mTOR and adenosine monophosphate (AMP) kinase (AMPK) can regulate the rate of autophagic vessel formation. ${ }^{14}$ Nucleation is controlled by a class 3 phosphatidylinositol-3-kinase (PI3K) called vacuolar protein sorting 34 (Vps34), which binds to a complex consisting of Atg6/Beclin-1, p150, and Atg14L. ${ }^{15}$ Inhibition of Vps34 with 3-methyl adenine (3-MA) or small interfering (si)RNA to Beclin-1 is commonly used as an experimental tool to inhibit autophagy. Maturation (elongation, curvature, and closure) is regulated via ubiquitin-like conjugation systems, which regulate LC3 (also known as Atg8/microtubule-associated protein 1 light chain 3 [LC3]-I/II). The first system generates LC3-II, which is the cleaved and lipidated (phosphatidylethonolamine [PE]) form of LC3 that is inserted into the autophagosomal membrane and often monitored by Western blot or immunocytochemistry as a marker for evaluating autophagy. The second system consists of Atg12 bound to Atg5 and Atg16L, which recruits LC3-II to the developing autophagosomal membrane. LC3 binding to the membranes is important for transport and maturation of the autophagosome, which later fuses its external membrane with lysosomes to degrade its cargo. LC3-II remains on mature autophagosomes until fusion with lysosomes is completed. LC3-II also binds to the adaptor protein p62/sequestosome-1 (SQSTM1), which is involved in trafficking proteins into the proteasome and serves to facilitate the autophagic degradation of ubiquitinated protein aggregates. P62/SQSTM1 is normally degraded during autophagy and accumulates when autophagy is impaired. Late events in autophagy involve the final maturation and fusion of autophagosomes with lysosomes to form an autolysosome, a step that requires small Rab GTPases and lysosome-associated membrane protein 2 (LAMP2).

Autophagy appears to play a significant role in the tumor microenvironment. The observation that coculture of cancer cells with fibroblasts results in reduced numbers of mitochondria in the fibroblasts and increased numbers of mitochondria in cancer cells has led to the "Reverse Warburg Effect" theory. ${ }^{13}$ This theory postulates that cancer cells induce a redox environment in the stroma, which induces mitophagy in the cancer-associated fibroblasts. The mitophagy releases glutamate from the fibroblast, which feeds the TCA cycle in cancer cells to efficiently produce 


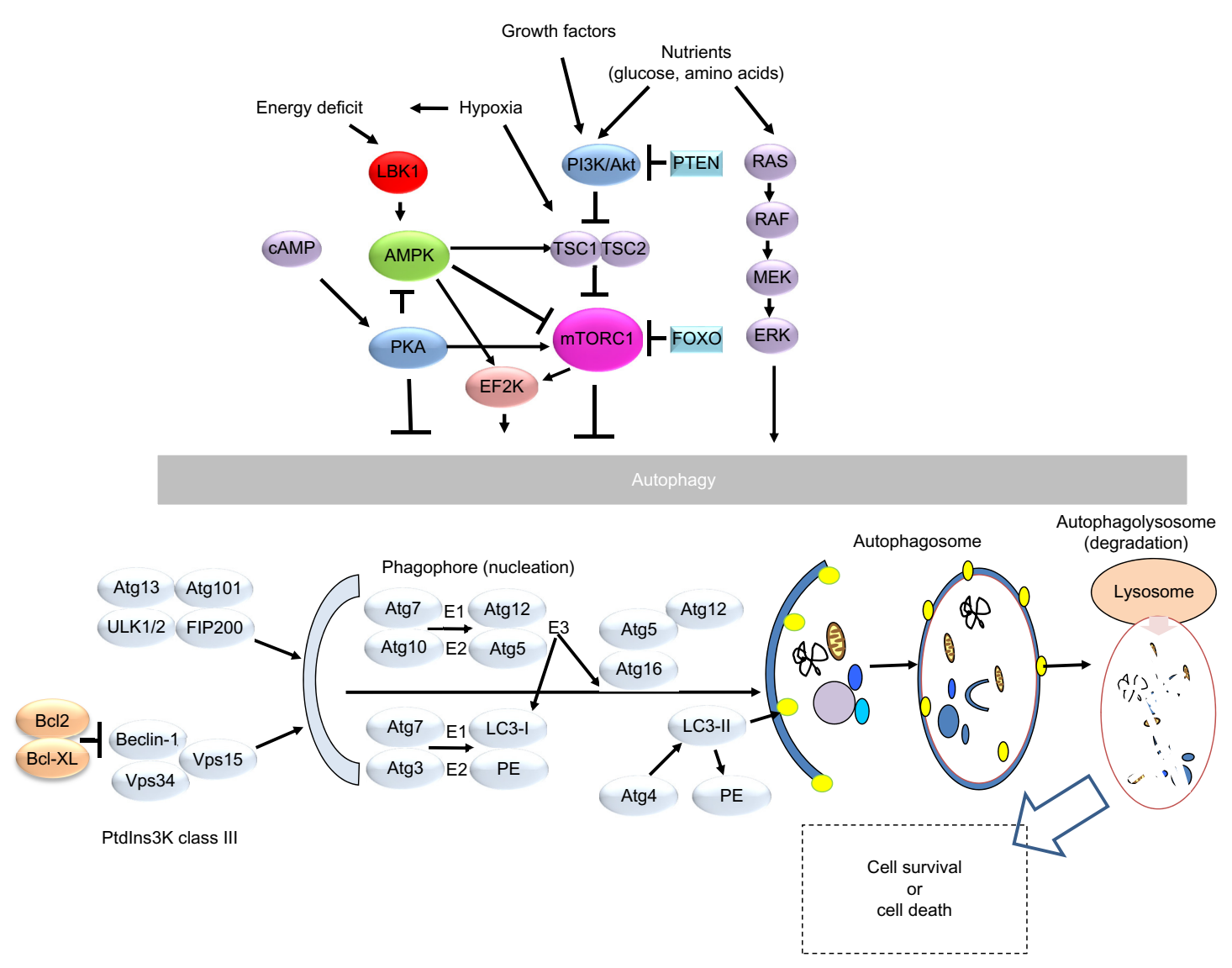

Figure I Regulation of autophagy.

Notes: $m$ TOR is one of the most important regulators of autophagy. mTOR and other pathways including cAMP, LKB, AMPK, and PKA merge at mTORCI. AMPK inhibits mTORCI by direct interaction or by indirect activation of the TSC2 protein. The mTORCI substrate p70S6K is a positive regulator of autophagy. Another important upstream factor is AKT/PKB, which acts a negative regulator of the TSCI/2 complex. In addition to energy depletion and hypoxia, the RAS, RAF, MEK, and ERK pathway is also involved in regulation of autophagy. The autophagic processes require induction, phagophore assembly (nucleation), sequestration, autophagosome formation, and autophagolysosome formation. The initial phase involves the initiation of the ULK complex, including ULKI/2, AtgI3, AtgI0I, and FIP200. The activation of the Ptdlns3K complex (Beclin-I, Vps34, and Vps I5), Vps, is an essential step in phagophore assembly (membrane nucleation). The EI-like enzyme Atg7 activates Atgl2 and LC3-I, and the E2-like enzymes AtglO (for activation of Atgl2) and Atg3 (for LC3-I). Atg5 is conjugated to the Atgl2 protein and this complex acts as an E3 ubiquitin ligase to catalyse the conjugation of LC3-I to PE in the process of sequestration. The subsequent autophagosome formation is dependent on the AtgI2-Atg5-Atg I6 complex. Once autophagosome formation is completed, the Atg I2-Atg5-Atg 16 complex dissociates from autophagosomes to allow Atg4 access to LC3-II for deconjugation from the lipid PE. Later, the lysosome merges with the autophagosome to form an autolysosome, which degrades the cytosolic macromolecules, proteins, and organelles. Depending on the cellular status, stress signal, and duration, the process leads to either cell death or cell survival.

Abbreviations: AKT/PKB, protein kinase B; mTOR, mammalian target of rapamycin; TAK, thylakoid membrane protein kinase; LKB, liver kinase B; AMPK, adenosine monophosphate kinase; PKA, protein kinase A; TOR, target of rapamycin; LC3, microtubule-associated protein I light chain; PE, phosphatidylcholine; cAMP, cyclic adenosine monophosphate.

adenosine triphosphate (ATP). A by-product of the TCA cycle, ammonia, released from the cancer cells continues to stimulate stromal cell mitophagy.

\section{Interpretation of autophagy markers}

"Guidelines for the use and interpretation of assays for monitoring autophagy" has recently been published in Autophagy by a group of autophagy experts under the leadership of $\mathrm{Dr}$ Daniel Klionsky. ${ }^{13}$ Although LC3-II expression, GFP-LC3 punctate formation, and transmission electron microscopy (TEM) are used commonly in in vitro studies, in clinical samples, autophagy is mostly evaluated by examining LC3-II and Beclin-1 expression by immunohistochemistry and autophagy genes by quantitative reverse-transcription polymerase chain reaction. ${ }^{13}$ Although TEM is the gold standard method to monitor autophagy, it is labor intensive; thus, evaluation of autophagy marker proteins in tissues using standard immunohistochemical techniques has become common practice. Increases in LC3-II levels are commonly used to detect the induction of autophagy in vitro and in vivo, but they can be misinterpreted if autophagy is blocked at the level of lysosomal fusion resulting in the buildup of autophagosomal vessels. To demonstrate autophagic flux, meaning autophagy that proceeds through to lysosomal degradation without blockage, inhibitors of lysosomal acidification and function, 
such as bafilomycin A, chloroquine (CQ), and hydroxychloroquine (HCQ), are commonly utilized in in vitro settings. ${ }^{13}$ Autophagic flux is confirmed if LC3-II is further increased in the presence of these inhibitors, but often, an additional molecule called p62 (also called SQSTM1) is evaluated to confirm this conclusion. The p62 protein binds to proteins that have been targeted for autophagosomal degradation by conjugation with single ubiquitin moieties or ubiquitin chains that are branched on Lys63, and that also, but with lower affinity, binds to proteins targeted for proteasomal degradation by conjugation with poly-ubiquitination chains that are branched on Lys48. ${ }^{17,18}$ The ubiquitinated proteins are then brought into the developing autophagosome by p62, which binds to LC3-II on the inner autophagosomal membrane. ${ }^{17,19}$ Upon fusion with the lysosome, p62 is degraded inside the autophagosome and, therefore, reduction of p 62 is considered a biomarker of autophagy. Prevention of p62 reduction with lysosomal inhibitors is considered an indication of autophagic flux. While these manipulations provide interpretable endpoints in tissue culture studies, total levels of p62 in tissue are less meaningful due to simultaneous induction of $\mathrm{p} 62$ by stresses that induce autophagy and the reduction of $\mathrm{p} 62$ by autophagic flux. ${ }^{20}$ Immunohistochemical analysis of either the A or B alleles of LC3, namely LC3A or LC3B, respectively, have been used to probe for the level of autophagy occurring in tissue. ${ }^{21,22}$ Although early studies suggested that only LC3B was involved in autophagy, more recent studies have indicated a role for $\mathrm{LC} 3 \mathrm{~A}$ in this process. ${ }^{23,24}$

While autophagy appears to engulf areas of the cytoplasm, specificity for individual molecules and organelles can be conferred by p62 and other molecules, such as Bcl-2 $19 \mathrm{kDa}$ interacting protein (BNIP3). In the presence of organelle damage, BNIP3 located on the cytoplasmic side of the mitochondria and ER membranes forms homodimers that have high affinity for LC3-II on the inside of autophagosomal membranes. ${ }^{25}$

\section{Autophagy as a tumor suppressor and cell survival mechanism}

An increasing body of evidence indicates that autophagy may be deregulated, suppressed, or overactivated in tumors. Whether autophagy is a prosurvival (oncogenic) process or a prodeath (tumor-suppressive) pathway is still controversial. ${ }^{26-32}$ However, studies indicate that this discrepancy depends on the cellular context (ie, mutations, genetic alterations), the status of activated or deactivated signaling pathways, and the extent of cellular stress. In breast cancer cells, Beclin-1 protein levels are often found to be low or undetectable, but high in all normal breast epithelial cells. ${ }^{33}$ Beclin-1 is monoallelically deleted in $40 \%-70 \%$ of cases of human breast, prostate, and ovarian cancers. ${ }^{33-36}$ Although biallelic mutations of Beclin-1 have not yet been demonstrated in human cancer cells, the monoallelic deletion of the Beclin-1 gene has also been observed in other cancer types, and autophagy is commonly inhibited in some of the aggressive tumors including ovarian and prostate cancer. ${ }^{37,38}$ The first evidence linking dysfunctional autophagy and cancer was demonstrated by a study where the disruption of Beclin-1 (BECN1) increased cellular proliferation and the frequency of spontaneous malignancies (ie, lung cancer, liver cancer, and lymphomas), as well as mammary hyperplasia, and accelerated the development of carcinogen-induced (ie, hepatitis B virus) premalignant lesions ${ }^{33}$ Furthermore, disruption of Beclin-1 resulted in reduced autophagy in mice, indicating that Beclin-1 is a haplo-insufficient tumor suppressor gene, providing evidence that autophagy is a novel mechanism of cell growth control and tumor suppression. These findings suggest that disruption of the function of Beclin-1 and other autophagy genes may contribute to the pathogenesis of human cancers. Later studies have demonstrated that defective autophagy leads to a reduction in degraded cellular contents, the induction of reactive oxygen species (ROS), DNA damage, genetic instability, and inflammation, providing conditions for transformation and carcinogenesis. ${ }^{35}$

The process of autophagy is thought to play a role in cancer initiation and progression, invasion and metastasis, and cancer stem cells and tumor dormancy. The induction of autophagy could lead to the survival of cancer cells in the highly metabolically challenged tumor microenvironment. In this environment, various stresses, including hypoxia, acidity (low $\mathrm{pH}$ ), increased metabolic products, reduced availability of nutrients due to high mitotic activity, and limited angiogenesis, activate autophagic scavenging of long-lived proteins and organelles to provide recycled components for reuse and repair. Extensive degradation by autophagy could lead to the degradation and loss of critical cellular components, which would cause a form of cell death (autophagic cell death), leading to the elimination of defective cells. ${ }^{4}$

\section{Signaling pathways involved in the regulation of autophagy}

A major regulator of autophagy is the mTOR pathway, downstream of protein kinase B (PI3K/AKT), which is often found to be overactivated in various human cancers. Excessive amino acids induce mTOR activity, which inhibits autophagy. High levels of AMP-active AMPK, which inhibits mTOR, 
induces autophagy. ${ }^{14}$ It is becoming clear that many signaling pathways and critical cellular proteins, including class 3 PI3K (Vps34), AMPK, Ras, Raf, MEK, ERK, JNK, p53, PTEN, p70S6K, eEF-2 kinase (EF2K), nuclear factor-kappa B (NF$\kappa \mathrm{B})$, and others, are involved in the regulation of autophagy. ${ }^{29}$ As previously stated, class 3 PI3K forms a complex with Beclin-1 and regulates nucleation. ${ }^{39-41}$ Both Bcl-2 and Bcl-XL antiapoptotic proteins can regulate autophagy by binding and inhibiting Beclin-1, and may calibrate autophagy to levels that are compatible with survival. ${ }^{42}$ Class $1 \mathrm{PI} 3 \mathrm{~K}$, the PI3K/AKT/ mTOR pathway, inhibits autophagy, whereas inhibitors of this pathway such as PTEN, TORCH1, AMPK, and rapamycin (a pharmacological mTOR inhibitor) induce autophagy. ${ }^{39,43}$ TOR has been shown to inhibit Atg1/ULK1. Rapamycin induces autophagy by restoring Atgl's kinase activity. ${ }^{44}$ The RAS/ RAF/MEK/ERK pathway can also promote autophagy. ${ }^{45}$ Recent findings suggest that NF- $\kappa \mathrm{B}$ inhibition can lead to an autophagic cell death. ${ }^{46,47}$ Tumor necrosis factor-alpha (TNF $\alpha$ ), insulin-like growth factor-1, and 7-ketocholesterol can regulate the expression of $A T G$ genes, including $L C 3$ and Beclin-1. In addition, p53 tumor suppressor protein is a transcription factor that responds to cellular stress and inhibits tumor formation. Recent studies also suggest that cytoplasmic p53 may inhibit autophagy, and that damage-regulated autophagy modulator (DRAM), a p53 target, can modulate autophagy ${ }^{48}$ Constitutive Ras activation induces autophagy to promote cell survival and tumor growth by altering mitochondrial function, underlying the role of Ras in tumors, which have mutated K-ras (ie, $90 \%-100 \%$ of pancreatic cancers). ${ }^{49}$ These observations represent critical findings regarding how signaling pathways control autophagy, and modulation of autophagy by targeting these pathways may be used as a therapeutic intervention depending on the cellular context.

\section{Targeting autophagy for cancer therapy and management}

Emerging data derived from in vitro and in vivo studies suggest that autophagy plays a critical role in maintaining cellular homeostasis and survival under stress conditions by eliminating damaged or malfunctioning organelles and toxic proteins, as well as by recycling building blocks and generating resources for ATP and energy. Autophagy is induced by various conditions in the harsh tumor microenvironment, including hypoxia (HIF-1 $\alpha$-dependent and -independent), metabolic stress, anticancer therapeutics, and radiation therapy. ${ }^{50-59}$ Thus, autophagy also plays a role in tumor cell survival and progression, as well as in response to anticancer therapies, which led to the hypothesis that the modulation of autophagy can be used as a therapeutic modality or can enhance the efficacy of cancer therapeutics. Currently, various clinical trials are investigating autophagy inhibitors in combination with cytotoxic chemotherapies and targeted agents in solid and hematological cancers (Table 1).

In vitro and in vivo studies demonstrated that the pharmacological inhibition (by HCQ, CQ, or 3-methyladenine [3-MA]) and genetic knockdown of autophagy genes augment the efficacy of various cancer therapeutics and targeted therapies. These studies led to the hypothesis that suppression of the autophagic pathway can be used as a sensitizing strategy for anticancer therapeutics. ${ }^{4,12,29}$ These include a number of antineoplastic therapies, DNA-damaging agents (eg, doxorubicin, temozolomide, etoposide), radiation therapy, histone deaceltylase (HDAC) inhibitors, suberoylanilide hydroxamic acid inhibitors, arsenic trioxide, TNF $\alpha$, interferon- $\gamma$, imatinib, rapamycin, and antiestrogen hormonal therapy (eg, tamoxifen), which have been shown to induce autophagy in some human cancer cell lines. ${ }^{50-59}$ In addition, recent data showed that human cancer cell lines with $H$-ras- or $K$-ras-activating mutations have high basal levels of autophagy and increased ROS levels, and that suppression of autophagy inhibits cell growth, indicating that autophagy is required for tumor cell survival, and that inhibition of this

Table I Clinical trials targeting autophagy in cancer patients

\begin{tabular}{lll}
\hline Tumor type & $\begin{array}{l}\text { Clinical } \\
\text { status }\end{array}$ & Therapeutic combination \\
\hline Advanced solid & Phase I & $\mathrm{HCQ}+$ temozolomide \\
tumor & Phase I & $\mathrm{HCQ}+$ sirolimus or vorinostat \\
& Phase I & $\mathrm{HCQ}+$ sunitinib \\
Metastatic melanoma & Phase I & $\mathrm{HCQ}+$ temsirolimus \\
Colorectal cancer & Phase II & $\mathrm{HCQ}+$ bevacizumab + XELOX \\
Glioblastoma & Phase I/II & $\mathrm{HCQ}+$ temozolomide + \\
multiforme & & radiation \\
Lung cancer & Phase I/II & $\mathrm{CQ}+$ cisplatin, etoposide \\
& Phase I/II & $\mathrm{HCQ}+$ carboplatin, paclitaxel \\
& Phase I/II & $\mathrm{HCQ}+$ bevacizumab \\
Prostate cancer & Phase II & $\mathrm{HCQ}+$ erlotinib \\
Breast cancer & Phase II & $\mathrm{HCQ}+$ docetaxel \\
& Phase II & $\mathrm{HCQ}$ only \\
& Phase I/II & $\mathrm{CQ}$ only \\
& Phase II & $\mathrm{CQ}+$ paclitaxel, docetaxel, \\
& ixabepilone \\
Renal cancer & Phase I & $\mathrm{HCQ}$ only \\
Pancreatic cancer & Phase I & $\mathrm{HCQ}$ only \\
& Phase I/II & $\mathrm{HCQ}+$ gemcitabine \\
Multiple myeloma & Phase I/II & $\mathrm{HCQ}+$ bortezomib \\
Leukemia (CLL) & Phase II & $\mathrm{HCQ}$ only \\
Leukemia (CML) & Phase II & $\mathrm{CQ}+$ imatinib \\
\hline Abbrevitions HCQ & &
\end{tabular}

Abbreviations: $\mathrm{HCQ}$, hydroxychloroquine; XELOX, capecitabine plus oxaliplatin; $\mathrm{CQ}$, chloroquine; CLL, chronic lymphocytic leukemia; CML, chronic myelogenous leukemia. 
process in Ras-driven cancers may be an effective treatment approach. ${ }^{61}$ In cancer cells that survive after chemotherapy and/or radiation therapy, autophagy induction leads to tumor dormancy in residual cancer cells that may contribute to tumor recurrence. ${ }^{60}$ Thus, targeting autophagy may prevent the dormant state and provide an effective strategy to induce better antitumor efficacy.

Clinical trials of autophagy inhibitors are using United States Food and Drug Administration (FDA)-approved antimalarial drugs such as HCQ and CQ, lysosmal inhibitors, which are known to inhibit autophagy (Table 1). The early results of some of these clinical studies indicate that autophagy inhibition in combination with anticancer therapies seem to be safe and can augment the efficacy of various anticancer therapies. ${ }^{62-66}$

One of the clinical trials evaluated whether blocking autophagy with HCQ enhanced cell death induced by alkylating chemotherapy in patients with advanced solid malignancies and melanoma (49 patients [73\%] had metastatic melanoma). ${ }^{62}$ These patients were given oral HCQ (200-1,200 mg daily) in combination with temozolimide (oral $150 \mathrm{mg} / \mathrm{m}^{2}$ daily) for $1-2$ weeks. This study evaluated the maximum tolerated dose (MTD), safety, pharmacokinetics, and pharmacodynamics of HCQ in combination with temozolomide. Patients tolerated the HCQ and temozolimide combination well with no dose-limiting toxicities. ${ }^{62}$ For the Phase II study, patients were given $600 \mathrm{mg}$ HCQ in combination with temozolomide twice daily, and some toxicities including grade 2 fatigue (55\% of patients), nausea (48\%), anorexia (28\%), constipation (20\%), and diarrhea (20\%) were observed. Overall, 3/22 (14\%) and 6/22 (27\%) patients with metastatic melanoma had partial responses and stable disease. ${ }^{62}$ Two out of six patients with refractory BRAF wild-type melanoma experienced a near complete response and prolonged stable disease. The study also evaluated and demonstrated the induction of autophagy in patient-derived peripheral blood mononuclear cells in response to combined therapy. Overall, the study indicated that the inhibition of autophagy by temozolomide may provide beneficial outcomes in melanoma patients.

The inhibition of autophagy has been shown to significantly augment the anticancer efficacy of the HDAC inhibitor, vorinostat. ${ }^{63}$ The combination of the autophagy inhibitor HCQ (taken orally on a daily basis from days 2-21 of a 21-day cycle) and vorinostat (400 mg, days 1-21) in patients with advanced solid tumors (27 patients) resulted in some side effects, including grade 1 to 2 nausea, diarrhea, fatigue, weight loss, anemia, and elevated creatinine. Fatigue and gastrointestinal side effects were the dose-limiting toxicities, and $600 \mathrm{mg}$ HCQ and $400 \mathrm{mg}$ vorinostat was established as the MTD and recommended Phase II regimen. In the treatment group, one patient with renal cell carcinoma had a durable partial response and two patients with colorectal cancer had prolonged stable disease. Currently, additional clinical studies are being conducted to further investigate the clinical significance of autophagy inhibition as a new strategy to enhance the efficacy of HDAC inhibitors.

Another preclinical study in multiple myeloma demonstrated that autophagy inhibition with HCQ significantly potentiates the efficacy of the proteasome inhibitor, bortezomib. ${ }^{75}$ In a Phase I study, ${ }^{64}$ a HCQ and bortezomib combination was evaluated in relapsed or refractory myeloma patients (25 patients; eleven (44\%) refractory to prior bortezomib treatment). Patients received HCQ (600 mg twice daily) with standard doses of bortezomib, ${ }^{51}$ and of 22 patients evaluable for response, three (14\%) had partial responses, three (14\%) had minor responses, and ten (45\%) had prolonged stable disease. The combined therapy showed therapy-associated increases in autophagy, and indicated the treatment's feasibility and potential benefits as a useful strategy for improving outcomes in myeloma patients.

Another Phase I study ${ }^{65}$ evaluated the safety and preliminary clinical activity of the HCQ and temsirolimus (an mTOR inhibitor) combination in patients with advance solid tumors, following preclinical demonstration that HCQ enhanced cell death in combination with temsirolimus (CCI-779). This dose escalation study evaluated the effects in an initial set of 27 patients, followed by a cohort of 12 patients with metastatic melanoma. Patients receiving the combination of HCQ and temsirolimus experienced grade 3 or 4 toxicity of anorexia (7\%), fatigue (7\%), and nausea (7\%). Although the MTD was not reached for HCQ, a Phase II study was conducted with a dose of $600 \mathrm{mg} \mathrm{HCQ}$ (twice daily) in combination with $25 \mathrm{mg}$ weekly of temsirolimus. Patients experienced grade 1 or 2 toxicities, such as rash, stomatitis, and weight loss. Overall, 14/21 (67\%) of all patients and 14/19 (74\%) of patients with melanoma achieved stable disease. Thirteen melanoma patients treated with HCQ 1,200 mg/day in combination with TEM had a median progression-free survival of about 3.5 months. Evaluation of peripheral blood monocytes and tumor biopsies demonstrated autophagy inhibition only in patients treated with the highest dose of $\operatorname{HCQ}(1,200 \mathrm{mg}$ daily). This study suggested that the combination of HCQ with temsirolimus was safe and well tolerated and, more importantly, it demonstrated significant antitumor activity and stable disease in $75 \%$ of cases of metastatic melanoma, 
indicating that autophagy inhibition is a feasible and promising strategy in these patients.

Another Phase I clinical study ${ }^{66}$ conducted among newly diagnosed glioblastoma (GB) patients evaluated the MTD and efficacy of HCQ in combination with radiation therapy and temsirolimus. Patients received HCQ (oral daily doses from $200-800 \mathrm{mg}$ ), radiation therapy, and temsiroliumus. The MTD for HCQ was $600 \mathrm{mg} /$ day in the three-regimen combination. The subsequent Phase II cohort (number $[\mathrm{n}]=76$ ) was also conducted, and in this study, patients had a median survival of 15.6 months. However, overall survival was not significantly improved.

Another randomized, double-blind, placebo-controlled study $^{67}$ for $\mathrm{GB}$ multiforme patients $(n=30)$ evaluated the benefits of adding CQ (150 mg/day) beginning on postoperative day 5 for 12 months to the treatments (chemotherapy or radiotherapy). CQ cotherapy seemed to improve midterm survival when given in addition to conventional therapy for GB multiforme. CQ cotherapy and radiation improved the response of brain metastasis with no increase in toxicity; however, CQ alone did not improve the response rate or overall survival. ${ }^{68}$ These results suggest that larger studies of CQ are needed to make firm conclusions, and that the efficacy can be achieved with consistent inhibition of autophagy with CQ, rather than with HCQ. In a clinical trial in dogs $(\mathrm{n}=30),{ }^{69} \mathrm{HCQ}$ and doxorubicin chemotherapy appeared to provide clinical benefit in non-Hodgkin's lymphoma with a $\sim 30 \%$ complete remission rate.

Overall, in vivo preclinical studies with animals and clinical trials with patients indicate that autophagy inhibitors may enhance the efficacy of conventional therapies in various cancers, including hematological cancers. However, larger clinical trials and a comprehensive analysis of the data will eventually provide more definitive answers regarding the effects of manipulation of authophagy in patients.

\section{Alternate approaches to inhibiting autophagy as a therapeutic strategy}

Because mTOR is a major negative regulatory axis for autophagy, several drugs that directly inhibit mTOR (rapamycin, temsirolimus, everolimus) and its pathways have been used to induce autophagy. The inhibition of mTOR mimics cellular starvation by blocking signals required for cell growth and proliferation. ${ }^{70}$ Some studies demonstrate that the PI3K/ $\mathrm{AKT} / \mathrm{mTOR}$ pathway promotes melanoma tumor growth and survival. ${ }^{70,71}$ Knockdown of the expression of the essential autophagy gene (ATG7) results in cell death, indicating that the survival of melanoma cells is autophagy-dependent.
Conversely, inhibition of mTOR with temsirolimus induces autophagy, which can promote tumor survival, and thus these agents may potentially limit their own efficacy. ${ }^{71}$ In support of this, inhibition of autophagy with HCQ synergizes with temsirolimus and leads to melanoma cell death via apoptosis. ${ }^{71}$ Combination treatments with temsirolimus and HCQ suppressed melanoma growth and induced cell death in both spheroid cultures and in tumor xenografts. ${ }^{71}$ These data suggest that inhibition of the mTOR and autophagy pathways promotes apoptosis and could be a new therapeutic paradigm for the treatment of melanoma. In addition, other strategies have been used to induce autophagy. For instance, knockdown of $\mathrm{Bcl}-2$ expression by siRNA induces autophagic cell death in breast cancer cell lines and in vivo in orthotopic xenograft models of ER(-) and ER(+) breast cancer. ${ }^{72,73}$ Also, targeting protein kinase $\mathrm{C}(\mathrm{PKC})$ delta and tissue transglutaminase, which inhibits autophagy in pancreatic cancer cells, can increase apoptosis. ${ }^{74}$ Inhibition of NF-kB, MAPK, JNK, P38, or the induction of ERK (another regulator of autophagy) has been used to modulate autophagy in different cancer models to alter responses to the various therapies, including chemo and radiation therapy. Overall, data suggest that the multiple and different strategies for the modulation of autophagy could improve therapy in some tumors, and that they can be utilized as cotherapy.

\section{Conclusion}

Recent findings suggested that autophagy is a promising target in the context of cancer therapy. Autophagy is thought to act as a prosurvival pathway that protects cancer cells from various stresses and anticancer therapy-induced stress, thus the inhibition of autophagy is considered in combination with some of the anticancer therapeutic strategies. The results of the first clinical trials conducted with autophagy inhibition along with conventional therapies indicate some clinical benefits in some cancers. Overall, these studies suggest that the efficacy of standard therapies may be enhanced when combined with autophagy inhibitors, which warrant further evaluation. Further understanding of the pathways regulating autophagy will likely offer new targets for the treatment of certain aggressive forms of tumors. Once the results of current clinical trials are obtained and analyzed, the role of autophagy will be better understood in tumors, and it would indicate whether modulation of the autophagic process or its related pathways that protect cancer cells from undergoing autophagy may be added to conventional therapies for better efficacy. In addition to currently available autophagy inhibitors, such as US FDA-approved malaria 
drugs (ie, HCQ, CQ), identification of novel, highly specific, and effective compounds to negatively regulate autophagy in cancer cells are needed.

\section{Disclosure}

The authors report no conflicts of interest in this work.

\section{References}

1. Yang Z, Klionsky DJ. Eaten alive: a history of macroautophagy. Nat Cell Biol. 2010;12(9):814-822.

2. Mizushima N, Yoshimori T, Ohsumi Y. The role of Atg proteins in autophagosome formation. Annu Rev Cell Dev Biol. 2011;27: $107-132$.

3. Codogno P. Shining light on autophagy. Nat Rev Mol Cell Biol. 2014;15(3):153.

4. Rubinsztein DC, Shpilka T, Elazar Z. Mechanisms of autophagosome biogenesis. Curr Biol. 2012;22(1):R29-R34.

5. Li WW, Li J, Bao JK. Microautophagy: lesser-known self-eating. Cell Mol Life Sci. 2012;69(7):1125-1136.

6. Fortun J, Verrier JD, Go JC, Madorsky I, Dunn WA, Notterpek L. The formation of peripheral myelin protein 22 aggregates is hindered by the enhancement of autophagy and expression of cytoplasmic chaperones. Neurobiol Dis. 2007;25(2):252-265.

7. Ashrafi G, Schwarz TL. The pathways of mitophagy for quality control and clearance of mitochondria. Cell Death Differ. 2013;20(1):31-42.

8. Kondo Y, Kanzawa T, Sawaya R, Kondo S. The role of autophagy in cancer development and response to therapy. Nat Rev Cancer. 2005;5(9):726-734.

9. Kroemer G, Jäättelä M. Lysosomes and autophagy in cell death control. Nat Rev Cancer. 2005;5(11):886-897.

10. Levine B, Kroemer G. Autophagy in the pathogenesis of disease. Cell. 2008;132(1):27-42.

11. Terman A, Gustafsson B, Brunk UT. Autophagy, organelles and ageing. J Pathol. 2007;211(2):134-143.

12. Wu WK, Coffelt SB, Cho CH, et al. The autophagic paradox in cancer therapy. Oncogene. 2012;31(8):939-953.

13. Klionsky DJ, Abdalla FC, Abeliovich H, et al. Guidelines for the use and interpretation of assays for monitoring autophagy. Autophagy. 2012;8(4):445-544.

14. Inoki K, Kim J, Guan KL. AMPK and mTOR in cellular energy homeostasis and drug targets. Annu Rev Pharmacol Toxicol. 2012;52: $381-400$.

15. Lindmo K, Stenmark H. Regulation of membrane traffic by phosphoinositide 3-kinases. J Cell Sci. 2006;119(Pt 4):605-614.

16. Yoon YH, Cho KS, Hwang JJ, Lee SJ, Choi JA, Koh JY. Induction of lysosomal dilatation, arrested autophagy, and cell death by chloroquine in cultured ARPE-19 cells. Invest Ophthalmol Vis Sci. 2010;51(11): 6030-6037.

17. Popovic D, Dikic I. The molecular basis of selective autophagy. Biochemist. 2012;34:24-30.

18. Ye Y, Shibata Y, Kikkert M, van Voorden S, Wiertz E, Rapoport TA. Recruitment of the p97 ATPase and ubiquitin ligases to the site of retrotranslocation at the endoplasmic reticulum membrane. Proc Natl Acad Sci U SA. 2005;102(40):14132-14138.

19. Wooten MW, Geetha T, Babu JR, et al. Essential role of sequestosome 1/p62 in regulating accumulation of Lys63-ubiquitinated proteins. J Biol Chem. 2008;283(11):6783-6789.

20. Puissant A, Fenouille N, Auberger P. When autophagy meets cancer through p62/SQSTM1. Am J Cancer Res. 2012;2(4):397-413.

21. Spowart JE, Townsend KN, Huwait $\mathrm{H}$, et al. The autophagy protein LC3A correlates with hypoxia and is a prognostic marker of patient survival in clear cell ovarian cancer. J Pathol. 2012;228(4): $437-447$.
22. Lazova R, Camp RL, Klump V, Siddiqui SF, Amaravadi RK, Pawelek JM. Punctate LC3B expression is a common feature of solid tumors and associated with proliferation, metastasis, and poor outcome. Clin Cancer Res. 2012;18(2):370-379.

23. Zois CE, Giatromanolaki A, Sivridis E, Papaiakovou M, Kainulainen H, Koukourakis MI. "Autophagic flux" in normal mouse tissues: focus on endogenous LC3A processing. Autophagy. 2011;7(11):1371-1378.

24. Giatromanolaki A, Koukourakis MI, Pouliliou S, et al. Overexpression of LC3A autophagy protein in follicular and diffuse large B-cell lymphomas. Hematol Oncol Stem Cell Ther. 2013;6(1):20-25.

25. Hanna RA, Quinsay MN, Orogo AM, Giang K, Rikka S, Gustafsson ÅB. Microtubule-associated protein 1 light chain 3 (LC3) interacts with Bnip3 protein to selectively remove endoplasmic reticulum and mitochondria via autophagy. J Biol Chem. 2012;287(23):19094-19104.

26. Kroemer G, Jäättelä M. Lysosomes and autophagy in cell death control. Nat Rev Cancer. 2005;5(11):886-897.

27. Ogier-Denis E, Codogno P. Autophagy: a barrier or an adaptive response to cancer. Biochim Biophys Acta. 2003;1603(2):113-128.

28. Scott RC, Juhász G, Neufeld TP. Direct induction of autophagy by Atg1 inhibits cell growth and induces apoptotic cell death. Curr Biol. 2007;17(1):1-11.

29. Dalby KN, Tekedereli I, Lopez-Berestein G, Ozpolat B. Targeting the prodeath and prosurvival functions of autophagy as novel therapeutic strategies in cancer. Autophagy. 2010;6(3):322-329.

30. Golstein P, Kroemer G. Cell death by necrosis: towards a molecular definition. Trends Biochem Sci. 2007;32(1):37-43.

31. Edinger AL, Thompson CB. Defective autophagy leads to cancer. Cancer Cell. 2003;4(6):422-424.

32. Tóth S, Nagy K, Pálfia Z, Réz G. Cellular autophagic capacity changes during azaserine-induced tumour progression in the rat pancreas. Up-regulation in all premalignant stages and down-regulation with loss of cycloheximide sensitivity of segregation along with malignant transformation. Cell Tissue Res. 2002;309(3):409-416.

33. Liang XH, Jackson S, Seaman M, et al. Induction of autophagy and inhibition of tumorigenesis by beclin 1. Nature. 1999;402(6762): 672-676.

34. Qu X, Yu J, Bhagat G, et al. Promotion of tumorigenesis by heterozygous disruption of the beclin 1 autophagy gene. J Clin Invest. 2003;112(12): 1809-1820.

35. Karantza-Wadsworth V, Patel S, Kravchuk O, et al. Autophagy mitigates metabolic stress and genome damage in mammary tumorigenesis. Genes Dev. 2007;21(13):1621-1635.

36. Saito H, Inazawa J, Saito S, et al. Detailed deletion mapping of chromosome $17 \mathrm{q}$ in ovarian and breast cancers: $2-\mathrm{cM}$ region on $17 \mathrm{q} 21.3$ often and commonly deleted in tumors. Cancer Res. 1993;53(14): 3382-3385.

37. Liang XH, Yu J, Brown K, Levine B. Beclin 1 contains a leucine-rich nuclear export signal that is required for its autophagy and tumor suppressor function. Cancer Res. 2001;61(8):3443-3449.

38. Gao X, Zacharek A, Salkowski A, et al. Loss of heterozygosity of the BRCA1 and other loci on chromosome 17q in human prostate cancer. Cancer Res. 1995;55(5):1002-1005.

39. Petiot A, Ogier-Denis E, Blommaart EF, Meijer AJ, Codogno P. Distinct classes of phosphatidylinositol 3'-kinases are involved in signaling pathways that control macroautophagy in HT-29 cells. J Biol Chem. 2000;275(2):992-998.

40. Mizushima N. Methods for monitoring autophagy. Int J Biochem Cell Biol. 2004;36(12):2491-2502.

41. Pattingre S, Tassa A, Qu X, et al. Bcl-2 antiapoptotic proteins inhibit Beclin 1-dependent autophagy. Cell. 2005;122(6):927-939.

42. Takacs-Vellai K, Vellai T, Puoti A, et al. Inactivation of the autophagy gene bec-1 triggers apoptotic cell death in C. elegans. Curr Biol. 2005; 15(16):1513-1517.

43. Arico S, Petiot A, Bauvy C, et al. The tumor suppressor PTEN positively regulates macroautophagy by inhibiting the phosphatidylinositol 3-kinase/protein kinase B pathway. J Biol Chem. 2001;276(38): 35243-35246. 
44. Takeuchi H, Kondo Y, Fujiwara K, et al. Synergistic augmentation of rapamycin-induced autophagy in malignant glioma cells by phosphatidylinositol 3-kinase/protein kinase B inhibitors. Cancer Res. 2005; 65(8):3336-3346.

45. Pattingre S, Bauvy C, Codogno P. Amino acids interfere with the ERK1/2-dependent control of macroautophagy by controlling the activation of Raf-1 in human colon cancer HT-29 cells. J Biol Chem. 2003;278(19):16667-16674.

46. Fabre C, Carvalho G, Tasdemir E, et al. NF-kappaB inhibition sensitizes to starvation-induced cell death in high-risk myelodysplastic syndrome and acute myeloid leukemia. Oncogene. 2007;26(28):4071-4083.

47. Mavrakis M, Lippincott-Schwartz J, Stratakis CA, Bossis I. Depletion of type IA regulatory subunit (RIalpha) of protein kinase A (PKA) in mammalian cells and tissues activates mTOR and causes autophagic deficiency. Hum Mol Genet. 2006;15(19):2962-2971.

48. Crighton D, Wilkinson S, Ryan KM. DRAM links autophagy to p53 and programmed cell death. Autophagy. 2007;3(1):72-74.

49. Guo JY, Karsli-Uzunbas G, Mathew R, et al. Autophagy suppresses progression of K-ras-induced lung tumors to oncocytomas and maintains lipid homeostasis. Genes Dev. 2013;27(13):1447-1461.

50. Rouschop KM, Wouters BG. Regulation of autophagy through multiple independent hypoxic signaling pathways. Curr Mol Med. 2009;9(4):417-424.

51. Kanzawa T, Germano IM, Komata T, Ito H, Kondo Y, Kondo S. Role of autophagy in temozolomide-induced cytotoxicity for malignant glioma cells. Cell Death Differ. 2004;11(4):448-457.

52. Paglin S, Hollister T, Delohery T, et al. A novel response of cancer cells to radiation involves autophagy and formation of acidic vesicles. Cancer Res. 2001;61(2):439-444.

53. Boya P, González-Polo RA, Casares N, et al. Inhibition of macroautophagy triggers apoptosis. Mol Cell Biol. 2005;25(3):1025-1040.

54. Qadir MA, Kwok B, Dragowska WH, et al. Macroautophagy inhibition sensitizes tamoxifen-resistant breast cancer cells and enhances mitochondrial depolarization. Breast Cancer Res Treat. 2008;112(3): 389-403.

55. Carew JS, Nawrocki ST, Kahue CN, et al. Targeting autophagy augments the anticancer activity of the histone deacetylase inhibitor SAHA to overcome Bcr-Abl-mediated drug resistance. Blood. 2007;110(1): 313-322.

56. Amaravadi RK, Yu D, Lum JJ, et al. Autophagy inhibition enhances therapy-induced apoptosis in a Myc-induced model of lymphoma. J Clin Invest. 2007;117(2):326-336.

57. Apel A, Herr I, Schwarz H, Rodemann HP, Mayer A. Blocked autophagy sensitizes resistant carcinoma cells to radiation therapy. Cancer Res. 2008;68(5):1485-1494.

58. Abedin MJ, Wang D, McDonnell MA, Lehmann U, Kelekar A. Autophagy delays apoptotic death in breast cancer cells following DNA damage. Cell Death Differ. 2007;14(3):500-510.

59. Katayama M, Kawaguchi T, Berger MS, Pieper RO. DNA damaging agent-induced autophagy produces a cytoprotective adenosine triphosphate surge in malignant glioma cells. Cell Death Differ. 2007;14(3): 548-558.

60. Lu Z, Luo RZ, Lu Y, et al. The tumor suppressor gene ARHI regulates autophagy and tumor dormancy in human ovarian cancer cells. J Clin Invest. 2008;118(12):3917-3929.
61. Guo JY, Chen HY, Mathew R, et al. Activated Ras requires autophagy to maintain oxidative metabolism and tumorigenesis. Genes Dev. 2011;25(5):460-470.

62. Rangwala R, Leone R, Chang YC, et al. Phase I trial of hydroxychloroquine with dose-intense temozolomide in patients with advanced solid tumors and melanoma. Autophagy. 2014;10(8):1369-1379.

63. Mahalingam D, Mita M, Sarantopoulos J, et al. Combined autophagy and HDAC inhibition: a phase I safety, tolerability, pharmacokinetic and pharmacodynamic analysis of hydroxychloroquine in combination with the HDAC inhibitor vorinostat in patients with advanced solid tumors. Autophagy. 2014;10(8):1403-1414.

64. Vogl DT, Stadtmauer EA, Tan KS, et al. Combined autophagy and proteasome inhibition: a phase 1 trial of hydroxychloroquine and bortezomib in patients with relapsed/refractory myeloma. Autophagy. 2014;10(8):1380-1390.

65. Rangwala R, Chang YC, Hu J, et al. Combined MTOR and autophagy inhibition: phase I trial of hydroxychloroquine and temsirolimus in patients with advanced solid tumors and melanoma. Autophagy. 2014;10(8):1391-1402.

66. Rosenfeld MR, Ye X, Supko JG, et al. A phase I/II trial of hydroxychloroquine in conjunction with radiation therapy and concurrent and adjuvant temozolomide in patients with newly diagnosed glioblastoma multiforme. Autophagy. 2014;10(8):1359-1368.

67. Sotelo J, Briceño E, López-González MA. Adding chloroquine to conventional treatment for glioblastoma multiforme: a randomized, double-blind, placebo-controlled trial. Ann Intern Med. 2006;144(5): 337-343.

68. Rojas-Puentes LL, Gonzalez-Pinedo M, Crismatt A, et al. Phase II randomized, double-blind, placebo-controlled study of whole-brain irradiation with concomitant chloroquine for brain metastases. Radiat Oncol. 2013;8:209.

69. Barnard RA, Wittenburg LA, Amaravadi RK, Gustafson DL, Thorburn A, Thamm DH. Phase I clinical trial and pharmacodynamic evaluation of combination hydroxychloroquine and doxorubicin treatment in pet dogs treated for spontaneously occurring lymphoma. Autophagy. 2014;10(8):1415-1425.

70. Jung CH, Ro SH, Cao J, Otto NM, Kim DH. mTOR regulation of autophagy. FEBS Lett. 2010;584(7):1287-1295.

71. Xie X, White EP, Mehnert JM. Coordinate autophagy and mTOR pathway inhibition enhances cell death in melanoma. PLoS One. 2013;8(1):e55096

72. Akar U, Chaves-Reyez A, Barria M, et al. Silencing of Bcl-2 expression by small interfering RNA induces autophagic cell death in MCF-7 breast cancer cells. Autophagy. 2008;4(5):669-679.

73. Tekedereli I, Alpay SN, Akar U, et al. Therapeutic silencing of Bcl-2 by systemically administered siRNA nanotherapeutics inhibits tumor growth by autophagy and apoptosis and enhances the efficacy of chemotherapy in orthotopic xenograft models of ER (-) and ER (+) breast cancer. Mol Ther Nucleic Acids. 2013;2:e121.

74. Ozpolat B, Akar U, Mehta K, Lopez-Berestein G. PKC delta and tissue transglutaminase are novel inhibitors of autophagy in pancreatic cancer cells. Autophagy. 2007;3(5):480-483.

75. Wu WK, Sakamoto KM, Milani M, et al. Macroautophagy modulates cellular response to proteasome inhibitors in cancer therapy. Drug Resist Updat. 2010;13(3):87-92.
Cancer Management and Research

\section{Publish your work in this journal}

Cancer Management and Research is an international, peer-reviewed open access journal focusing on cancer research and the optimal use of preventative and integrated treatment interventions to achieve improved outcomes, enhanced survival and quality of life for the cancer patient The journal welcomes original research, clinical \& epidemiological

\section{Dovepress}

studies, reviews \& evaluations, guidelines, expert opinion \& commentary, case reports \& extended reports. The manuscript management system is completely online and includes a very quick and fair peerreview system, which is all easy to use. Visit http://www.dovepress.com/ testimonials.php to read real quotes from published authors. 